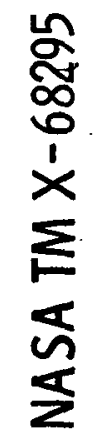

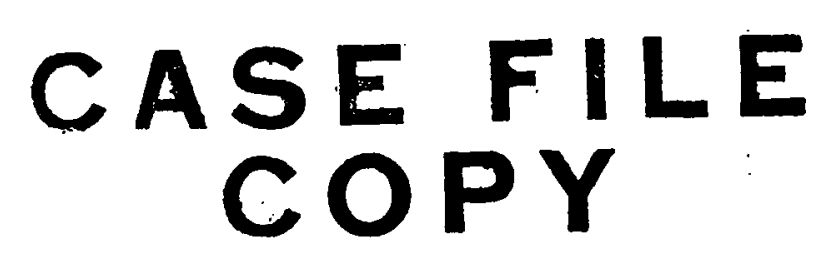

\title{
A DIFFERENCE THEORY FOR NOISE PROPAGATION IN AN ACOUSTICALLY LINED DUCT WITH MEAN FLOW
}

by Kenneth J. Baumeister and Edward J. Rice

Lewis Research Center

Cleveland, Ohio 44135

TECHNICAL PAPER proposed for presentation at Aero-acoustic Specialists Conference sponsored by the American Institute of Aeronautics and Astronautics Seattle, Washington, October 15-17, 1973 


\section{A DIFFERENCE THEORY FOR NOISE PROPAGATION IN AN ACOUSTICALLY LINED DUCT WITH MEAN FLOW}

Kenneth $J$. Baumeister and Edward J. Rice National Aeronautics and Space Administration Lewis Research Center Cleveland, ohio

\section{Abstract}

A finite difference formulation is presented for sound propagation in a two-dimensional straight soft-walled duct wi th uniform flow. The difference analysis is developed in terms of complex notation. The governing acoustic difference equations and the appropriate displacement boundary conditions associated with uniform flow are presented. Example calculations are presented for the sound at tenuation in straight hard and soft-walled ducts. At present the finite Mach number case is solved only for the one-dimensional hard walled duct.

\section{Introduction}

In the aircraft industry, noise suppressors are now an integral part of turbofan engine installation design. In particular; suppressors must be optimized for noise at tenuation wi th minimum weight, length, and total pressure loss. Presently, estimates for the attenuation of acoustic energy (noise) in a suppressor are based on experimental data or on analytical studies $(1-7)$ of acoustic waves propagating into infinitely long straight ducts with uniform impedance (absorbers) along the walls. The analytical techniques, however, are limited to relatively simple geometries.

There is a need for more flexible suppressor design techniques which can handle acoustical flow field complications arising from (1) axial variations in Mach number, (2) axial variations in wall impedance associated with the effect of Mach number on the acoustical properties, or (3) self-generated noise sources at the boundary. To meet these needs, the present paper develops a numerical.finite difference technique for uniform mean flow. This technique represents an extension of a difference theory developed for noise propagation in ducts wi thout steady flow. (8) The addition of a uniform Mach number flow complicates the previous no-flow theory by the introduction of convective terms in both the governing equations and boundary conditions.

The governing acoustic difference equations and the appropriate displacement boundary conditions associated with uniform flow are presented. Immediately following the mathematical development of the difference technique, numerical solutions are presented for one and two dimensional hard and sof $t$ walled ducts. The results are compared with the corresponding analytical results.

\section{List of Symbols} speed of sound

$d B$ decibels, Eq. (26) acoustic power, Eq. (25)

f frequency

H channel height, see fig. 1

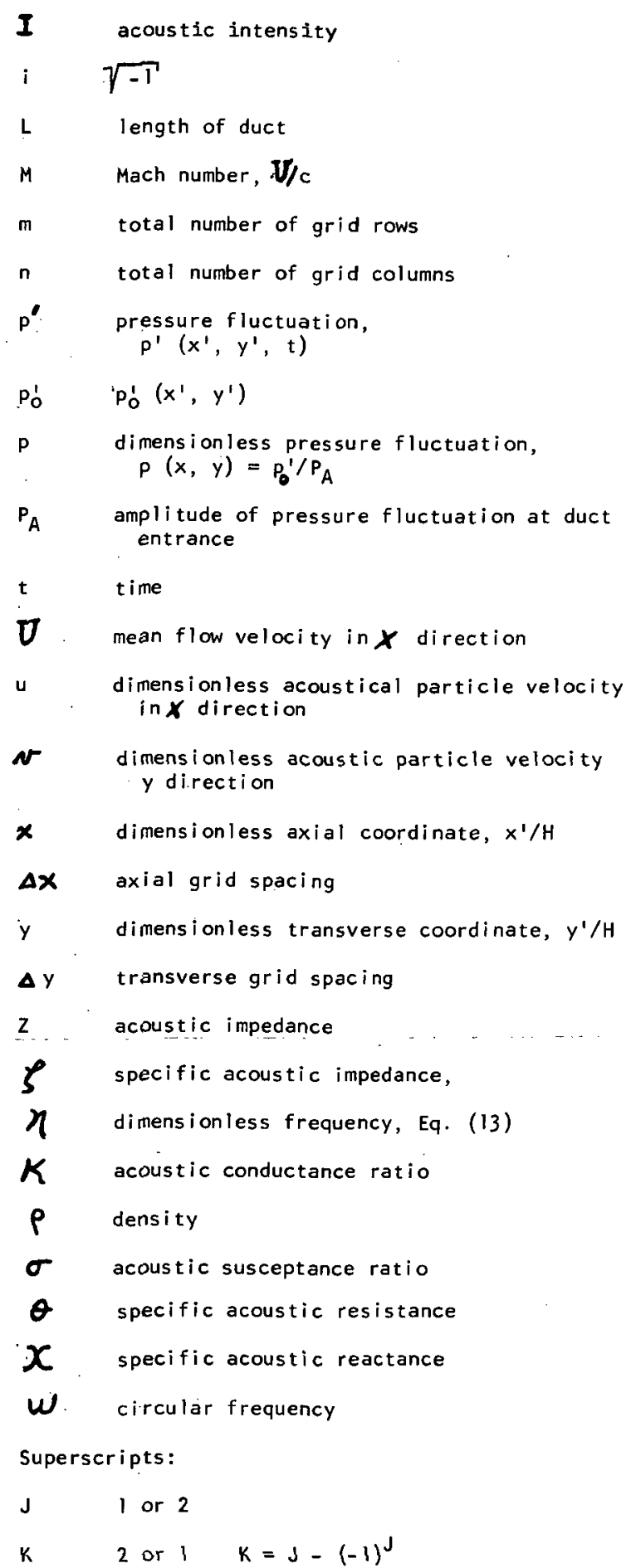




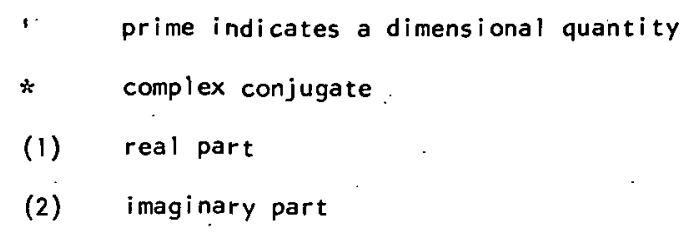

Subscripts:

exit condition
$i, j \quad \begin{aligned} & \text { axial index, } j \text { transverse index, } \\ & \text { see fig. } 2\end{aligned}$
wall
$x \quad$ axial position
$y \quad$ transverse position

Governing Equationa and Boundary Conditions

The propagation of sound in a two dimensional rectangular duct, as shown in Figure la, is described by a solution of the continuity and momentum equations with the appropriate impedance boundary conditions. In this section, the basic governing equations and boundary conditions will first be nondimensionalized to show the important property groups. Next, the difference form of the governing equations and boundary conditions will be developed.

\section{Continuity and Momentum}

The linearized equations for mass and momentum conservation can be written for a cartesian coordinate system in the following form:

$$
\begin{gathered}
\text { (continuity) } \\
\frac{\partial p^{\prime}}{\partial t}+v \frac{\partial p^{\prime}}{\partial x}+\rho c^{2}\left(\frac{\partial u^{\prime}}{\partial x^{\prime}}+\frac{\partial w^{\prime}}{\partial y}\right)=0 \\
\text { (x-momentum) } \\
\frac{\partial u^{\prime}}{\partial t}+v \frac{\partial u^{\prime}}{\partial x}=-p \frac{\partial p^{\prime}}{\partial x^{\prime}} \\
(v \text {-momentum) } \\
\frac{\partial w^{\prime}}{\partial t}+v \frac{\partial v^{\prime}}{\partial x^{\prime}}=-\frac{1}{\rho} \frac{\partial p^{\prime}}{\partial y^{\prime}}
\end{gathered}
$$

The assumptions involved in the derivations of Equation (1) through (3) are given in most acoustic texts and will not be discussed herein. The single prime, ', is used to denote dimensional quantities. It will be removed when the equations are made dimensionless. All the symbols used in this report are defined in the list of symbols.

As customary for steady state, the solutions for the dependent pressures and velocities are assumed to be of the form

$$
\begin{aligned}
& P^{\prime}\left(x ; y^{\prime}, t\right)=g^{\prime}\left(x^{\prime} ; y^{\prime}\right) e^{i \omega t} \\
& u^{\prime}\left(x ; y^{\prime}, t\right)=u_{0}^{\prime}\left(x^{\prime} ; y^{\prime}\right) e^{i \omega t} \\
& v^{\prime}\left(x ; x^{\prime}, t\right)=v_{0}(x ; y) e^{i \omega t}
\end{aligned}
$$

The final solution will be represented by the real part of the above quantities. Substitution of
Equations (4) through (6) into Equation (1) through (3) and introduction of the following dimensionless parameters

$$
\begin{array}{ll}
p=P_{0}^{\prime} \%_{A} & \\
u=\frac{\rho \omega H}{H} u_{0}^{\prime} & v=\frac{\rho \omega H}{\dot{H}_{A}} N_{0}^{\prime} \\
x=\frac{x^{\prime}}{H} & y=\frac{y^{\prime}}{H}
\end{array}
$$

yield the dimensionless steady state conservation equations

$$
\begin{aligned}
& p-\frac{i m}{2 \pi} \frac{\partial p}{\partial x}-\frac{i}{(2 \pi n)^{2}}\left(\frac{\partial u}{\partial x}+\frac{\partial v}{\partial y}\right)=0 \\
& u=i \frac{\partial p}{\partial x}+i \frac{M}{2 m} \frac{\partial u}{\partial x} \\
& v=i \frac{\partial p}{\partial y}+i \frac{\mu}{\partial \pi} \frac{\partial \mu}{\partial x}
\end{aligned}
$$

The dimensionless frequency $\boldsymbol{M}$ is given as

$$
\eta=\frac{\mu}{2 \pi} \frac{\omega}{c}=\frac{H f}{c}
$$

For practical linear design, $\boldsymbol{\eta}$ is on the order of unity, which will lead to a manageable grid size in the numerical representation.

The above dimensionless equations apply to the scaled cartesian coordinate system shown in figure l(b). The dimensionless height $y$ ranges between zero and 1 while the dimensionless length $x$ ranges between zero and $L / H$. The solution of Equations (10) through (12) with the appropriate boundary conditions will lead to the determination of the duct at tenuation.

\section{Wave Equation}

Equations (10) through (12) are now combined to yield the dimensionless wave equation for uniform flow

$$
\left(i-m^{2}\right) \frac{)^{2} p}{\partial x^{2}}+\frac{y^{2}}{\partial y^{2}}+(2 \pi x)^{2} p-i 4 \pi n M \frac{\partial p}{\partial x}=0
$$

Equation (14) in difference form will be solved to determine the pressure in the düct.

When using the exponential notation displayed in Equations (4) through (6), the dimensionless pressure and velocities have in general both real and imaginary parts. Thus,

$$
\begin{aligned}
& P(x, y)=p^{(1}(x, y)+i p^{(2)}(x, y) \\
& u(x, y)=u^{(2)}(x, y)+i u^{(2)}(x, y) \\
& v(x, y)=v^{(1 y}(x, y)+i v^{(2)}(x, y)
\end{aligned}
$$

Consequently, Equation (14) can be broken into its real and imaginary parts by substituting Equation (15) into Equation (14). The resulting two equations can be represented as a single equation 


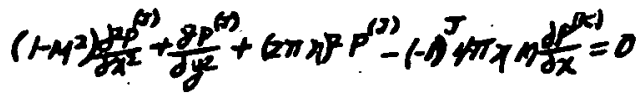

where $J$ equals 1 or 2 and $K$ equals 2 or 1 , respectively. The two equations represented by Equation (18) represent the basic governing equation for noise propagation which will be solved later by a difference formulation. As can be seen in Equation (18), the real pressure $p(1)$ is coupled to the imaginary pressure $p(2)$ through the term containing Mach number to the first power. For zero Mach number, $p(1)$ and $p(2)$ will be coupled by the impedance conditions along the walls.

Whenever the $J$ and $K$ symbols are used later in this paper, as in Equation (18), the single equation should be interpreted as representing two equations containing the real and imaginary contributions.

\section{Displacement Boundary Condition}

The boundary condition at the surface of a soft-wall duct requires that at the wall the acoustic displacement of a particle in the fluid be equal to the displacement of a particle just inside the soft wall. (5) In addition, the pressure and velocity fields at the boundary can now be related in terms of the specific acoustic impedance $\boldsymbol{f}$, where

$$
S=\frac{2 \pi X_{P}}{N}
$$

As a consequence of these two relationships, it can be shown that the appropriate boundary condition for slip flow is

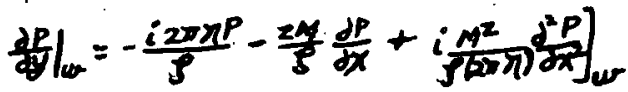

It is convenient to express the reciprocal of the impedance ratio $f$ in terms of the conductance ratio $K$ and the acoustic susceptance ratio

$$
\sigma \text {, that is, }
$$

$$
\frac{1}{\zeta} \equiv K-i \sigma
$$

where $1 / 9$ is the acoustic admittance ratio. Substituting Equations (15) and (20) into Equation (19) yields

$$
\begin{aligned}
& \left.\frac{\partial p^{(J)}}{\partial y}\right]_{v}^{d s}=-\left[2 \pi x \left[\sigma p^{(J)}+\left(-\partial^{\sigma} X p^{(0)}\right]_{v}\right.\right. \\
& -2 M\left[K \frac{\partial P(j)}{\partial x}-(-1)^{J} \sigma \frac{\partial p^{(}(k)}{\partial x}\right]_{\omega} \\
& +\frac{M^{2}}{2 \pi n}\left[\sigma \frac{\partial^{2} p(s)}{\partial x^{2}}+(-1)^{\top} K \frac{\partial p^{(x)}}{\partial x^{2}}\right]_{w}
\end{aligned}
$$

Equation (21 represents the boundary condition on pressure to be used at the treated surfaces of the duct.

\section{Entrance Conditions}

For the entranfe pressure profile, the assumption used by Rice (1) of a uniform profile will be used herein. At the present time, there is insufficient information available to improve on this assumption. For a uniform pressure profile, as will be used in the example considered later,

$$
P^{(1)}(0, y)=1 \quad P^{(2)}(0, y)=0
$$

A more general entrance condition where $p^{(1)}$ depends on $y$ is equally easy to treat.

\section{Exit Boundary Conditions}

Finally, the impedance must be specified at either the duct exit or at some point in the far field. The particular exit condition used in the example problem presented at the end of this paper will be discussed in a later section.

\section{Axial Acoustic Power}

The sound power which leaves a duct and reaches the far field is related to the axial intensity at the duct exit which for uniform flow is given in reference 1 as

$$
\begin{aligned}
& I^{\prime}=\frac{1}{z} \operatorname{REAL}\left\{P^{\prime \prime} u^{\prime}+\frac{M}{L}\left[\frac{p^{\prime \prime} p^{\prime}}{p^{C}}\right.\right. \\
& \left.\left.+\rho c\left(u^{2} u+w^{*} v\right)\right]\right\}
\end{aligned}
$$

Substitution of Equations (15) through (17) into Equation (23), and non-dimensionalizing the resulting equat $i$ on yeilds

$$
\begin{aligned}
& I=\frac{2 p c}{R^{2}} I^{\prime}=\frac{l}{2 m n}\left[p^{(1)} u^{(1)}+p^{(2)} u^{(t)}\right] \\
& +\frac{M}{2}\left[p^{()^{2}}+p^{(2)^{2}}\right] \\
& +\frac{M}{2(2 \pi n)^{2}}\left[a^{(1)^{2}}+n^{(2)^{2}}+N^{(2)^{2}}+w^{(2)^{2}}\right]
\end{aligned}
$$

The total dimensionless acoustic power is the integral of the intensity across the test section

$$
E_{x}=\int_{0}^{\prime} I(x, y) d y
$$

By definition, the sound attenuation (the decrease in decibels of the acoustic power from $x=0$ to $x$ ) can be written as

$$
\begin{aligned}
& \Delta d B=10 \log _{0}\left[\frac{E_{x}}{E_{0}}\right] \\
& \text { Finite Difference Formulation }
\end{aligned}
$$

Instead of a continuous solution for pressure the pressure will be determined at isolated grid points by means of the finite difference approximations, as shown in Figure 2. This enables changing the differential equations to a system of algebraic equations for the pressure and velocity at each grid point. The governing equations and boundary conditions can be approximated in difference form $(9)$ by using either a Taylor series expansion, a variational, or integral formulation. In this acoustic problem, where the gradient is specified along a boundary, the integration method for generating the $f i$ nite difference approximations is most convenient.

\section{Wave Equation}

The wave equation in finite difference form is developed by applying the integration method (ref. 9, p. 168) to the cell marked no. I in Figure 2. That is, the wave equation, equation (18), is integrated over the entire area of the cell marked number 1 in Figure 2. The details necessary for the development of the difference equations are given in reference 9 as well as many other texts; consequently, only the final results are now presented. The difference 
form of Equation (18) becomes:

$$
\begin{aligned}
& -\left(\frac{\Delta x}{\Delta y}\right)^{2} p_{i, j-1}^{(J)}-\left(1-M^{2}\right) p_{i-1, j}^{(J)} \\
& +2\left[1-M^{2}+\left(\frac{\Delta x}{\Delta y}\right)^{2}-\frac{(2 \pi n \Delta x)^{2}}{2}\right] p_{i, j}^{(J)} \\
& -\left(1-M^{2}\right) p_{i+t, j}^{(j)}-\left(\frac{\Delta x}{\Delta y}\right)^{2} P_{i, j+1}^{(J)} \\
& -(-1)^{J} 2 \pi n M \Delta x p_{i-j, j}^{(x)} \\
& +(-1)^{J} 2 \pi \eta M \Delta x p_{i+1, j}^{(k)}=0
\end{aligned}
$$

\section{Displacement Boundary Condition}

Along the upper and lower boundaries, cell no. 2 , the wave equation. Equation (18), is again integrated, only this time Equation (21) is involved in the integration along the boundary. The difference equation that applies in cell no. 2 is

$$
\begin{aligned}
& -\left(\frac{\Delta x}{\Delta y}\right)^{2} p_{i, m-1}^{(J)} \\
& -\left[\frac{1}{2}\left(1-M^{2}\right)+M K \frac{\Delta x}{\Delta y}+\frac{M^{2} \sigma}{2 \pi / \Delta y}\right] P_{\dot{\sigma}, m}^{(J)} \text {. } \\
& +\left[1-M^{2}+\left(\frac{\Delta x}{\Delta y}\right)^{2}-\frac{(2 \pi x \Delta x)^{2}}{2}+2 \pi \eta \sigma \frac{\Delta x^{2}}{\Delta y}\right. \\
& \left.+\frac{2 M^{2} \sigma}{2 \pi r \Delta y}\right] p_{i m}^{r n} \\
& -\left[\frac{1}{2}\left(1-M^{2}\right)-M K \frac{\Delta X}{\Delta y}+\frac{M^{2} \sigma}{2 \pi \eta} \Delta y\right] P_{i+1, m}^{(J)} \\
& -(-1)^{J}\left[\frac{2 \pi r \Delta x M}{2}-M \sigma \frac{\Delta x}{\Delta y}+\frac{M^{2} x}{2 \pi x \Delta y}\right] P_{i-1, m}^{(t)} \\
& +(-1)\left[2 \pi x K \frac{\Delta x^{2}}{\Delta y}+\frac{2 M^{2} x}{2 \pi x \Delta y}\right] P_{L, m}^{(k)} \\
& \begin{array}{c}
-(-1)^{\top}\left[\frac{-2 \pi M M \Delta x}{2}+M \sigma \frac{\Delta x}{\Delta y}+\frac{M^{2} K}{2 \pi x \Delta y}\right] P_{i+b m}^{(K)} \\
=0
\end{array} \\
& \text { Exit Condition }
\end{aligned}
$$

The exit condition to be presented now will allow the numerical solutions to be compared to analytical results for wave propagation in an infinitely long duct. The entrance region of length $L$ in an infinitely long duct with uniform impedance will not have reflections at any position in the duct. The wave propagation in the entrance region of the infinite duct can be represented with a finite length of duct $L$ by closing the exit impedance at $L$ so that no reflection would occur. Consequently, if the numerical exit condition in the numerical analysis is chosen to eliminate reflections at the exit of the duct, the numerical and analytical results should be in close agreement.

For a plane wave propagating in a hard-wall duct, the condition for no reflections at the duct exit is an exit impedance of PC which leads through the momentum equation to, the following exit condition

$$
\left.\left.\frac{\partial P}{\partial x}\right)_{e}=\frac{-i 2 \pi n}{(1+M)} P\right)_{e}
$$

In terms of the real and imaginary pressures, this equation becomes

$$
\left.\left.\frac{\partial P^{(J)}}{\partial x}\right)_{e}=\frac{-(-1)^{J}(2 \pi \mu)}{(1+M} P^{(k)}\right)_{e}
$$

We.will use Equation (30) as the boundary condition at the duct exit. Using Equation (30), the difference representation of Equation (18) at the duct exit (cell no. 3) becomes

$$
\begin{aligned}
-\frac{1}{2} & \left(\frac{\Delta x}{\Delta y}\right)^{2} P_{m, j-1}^{(J)}-\left(1-M^{2}\right) p_{m-1, j}^{(J)} \\
+ & {\left[1-M^{2}+\left(\frac{\Delta x}{\Delta y}\right)^{2}-\frac{(2 \pi n \Delta x)^{2}}{2}\right] P_{m, j}^{(J)} } \\
- & \frac{1}{2}\left(\frac{\Delta x}{\Delta y}\right)^{2} p_{m, j+1}^{(\sigma)} \\
& -(-1)^{J} 2 \pi r M \Delta x P_{m-1, j}^{(K)} \\
& +(-1)^{J} z \pi r \Delta x P_{m, j}^{(N)}=0
\end{aligned}
$$

Further details on the theory and limitations of Equation ( 31 ) are given in reference 8 .

\section{Corner Condition}

At cell no. 4 in Figure 2, we apply the same integration technique and get a difference equation similar to the other equations presented.

\section{Acoustic Particle Velocity}

Recall from Equation (24) that the intensity is a function of the acoustic particle velocities. We must solve the two momentum equations, Equation (11) and (12) for the velocities. In terms of the real and imaginary terms, Equations (11) and (12) can be rewritten as

$$
u^{(\pi)}=\left[\frac{\partial p^{(k)}}{\partial x}+\frac{M}{2 \pi r} \frac{\partial u^{(k)}}{\partial x}\right](-1)^{J}
$$

$\nu^{(J)}=\left[\frac{\partial P^{(\alpha)}}{\partial y}+\frac{M}{2 \pi n} \frac{\partial u^{(\alpha)}}{\partial y}\right](-1)^{J}$ 
where the condition of irrotationality

dy $=$ dy

was used in developing equation (33).

Since the pressure gradients in the above equations can be obtained directly from a numerical solution to the wave equation, Equations (32) and (33) can be treated as ordinary differential equations. As a result, a standard fourth-order Runge-Kut ta integration is used for the solution of equation (32). The solution of Equation (33) can be found by a simple differentiation process once $u$ is known.

The initial conditions for the Runge-Kutta solution can be found directly from an earlier part of the present work. In developing the displacement boundary condition, it also can be shown that the velocity at the wall is

$$
N)_{\omega}=\frac{2 \pi r}{\zeta}\left[P-\frac{i M}{2 \pi r} \frac{\partial P}{\partial x}\right]_{u^{r}}
$$

In a similar manner, the $u$ velocity at the exit can be found directly from the exit impedance relationship

$$
\left.u_{e}=2 \pi n P\right)_{e}
$$

\section{Axial Intensity}

In terms of the difference notation, the axial intensity as given by Equation (24) can be expressed as

$$
\begin{aligned}
I_{i, j}= & \frac{1}{2 \pi x}\left[p_{i, j}^{(1)} u_{i, j}^{(1)}+p_{i, j}^{(2)} u_{i, j}^{(2)}\right] \\
& +\frac{M}{2}\left[p_{i, j}^{(1)^{2}}+p_{i, j}^{(2) 2}\right] \\
& +\frac{M}{2(2 \pi n)^{2}}\left[u_{i, j}^{(1)^{2}}+u_{i, j}^{(2)^{2}}\right. \\
& \left.+N_{i, j}^{(1) 2}+N_{i, j}^{(2)^{2}}\right]
\end{aligned}
$$

The total power across a particular cross section, as given by Equation (25), is written in difference notation as

$$
E_{i}=\left[\frac{1}{2} I_{i, 1}+\sum_{j=2}^{m-1} I_{i, j}+\frac{1}{2} I_{i, n}\right] \Delta y
$$

By evaluating $\boldsymbol{E}_{\boldsymbol{i}}$ at the entrance and exit positions, taking the log of their ratio and multiplying by 10 , as indicated in Equation (26), the sound attenuation for the duct is determined.

\section{Matrix Solution}

The collection of the various difference equations at each grid point forms a set of simultaneous equations which can be expressed in matrix notation as

$$
\left[\begin{array}{c:c}
A & -C \\
\hdashline C & A
\end{array}\right]\left[\begin{array}{c}
p^{(1)} \\
\hdashline p^{(z)}
\end{array}\right]=\left[\begin{array}{c}
F^{(1)} \\
\hdashline F^{(2)}
\end{array}\right]
$$

where the A submatrix has a form typical of those matrices found in two-dimensional heat conduction problems, while the $C$ matrix represents the coupling that occurs between the $p(1)$ and $p(2)$ pressures through both the governing differential equations and impedance boundary conditions. The $C$ matrix is a tridiagonal matrix. The $F$ column vector contains the initial conditions.

The frequency term $(2 \pi x \Delta x)^{2}$ in equation (27) is a subtractive term in the main diagonal element of the coefficient matrix; consequently, for sufficiently high frequency or spacing parameters, the matrix will no longer be positive definite (ref. 10, pg. 68). As a result, conventional iteration techniques can not be used. However, matrices of the form of Equation (38) can be solved by elimination techniques. In particular, the Gauss el imination technique will be used to find a solu$t i$ on to the example problems which now follow. At present, the finite difference technique can not be applied to problems which require a large number of grid points because a subroutine based on a square full matrix is used for the solution. This subroutine requires large amounts of computer storage. iteration techniques or more efficient closed form solutions will have to be developed to overcome present grid size limitations.

\section{Example Calculations}

Example calculations are presented in this sec$t i o n$ so that a comparison between the finite difference solutions and corresponding analytical solutions can be made. First, some solutions for wave propagation with no mean flow (zero Mach number) are presented. Next, solutions for wave propagation in a hard wall duct wi th uniform flow are presented.

\section{Zero Mach Number}

Hard wall duct. Numerical and analytical values of the pressure are computed for the case of a one dimensional hard wall duct with a pC exit impedance. Because there is no variation of pressure in the $y$ direction, the two dimensional grid lattice shown in figure 2 reduces to a one-dimensional lattice as shown in the upper sketch of figure 3 . The calculation was made for a hard wall $(Z=\infty)$ with an $\mathrm{L} / \mathrm{H}$ of $\mathrm{I}$; an inlet plane wave with a dimensionless frequency $\boldsymbol{y}$ equal to 1 , and zero Mach number The analytical and numerical values of the acoustic pressure profiles along the duct are shown in Figure 3. As seen in Fiqure 3, agreement between the numerical and analytical results is good.

Soft wall duct. As another example of the finite difference formulation, the noise at tenuation will now be calculated for a two-dimensional duct with a dimensionless frequency $\pi$ of 0.6 , an $L / H$ of 0.5 , and a finite value of wall impedance. At the present time for two dimensional solutions, the technique is limited to $\mathrm{L} / \mathrm{H}$ values of 0.5 or less because of matrix size limitations discussed previously. 
Sound attenuation values were obtained by caiculating the appropriate values of $K$ and $\sigma$ from Equation (20), solving the matrix equation (38) by Gauss elimination for the pressure distribution, solving Equations (32) and (33) for the velocity distribution, and finally calculating the sound attenuation at the exit from Equations (37) and (26)

Because of the matrix size limitations in the subprogram used to solve Eqyation (38), the technique of varying grid size (12) was used. This is illustrated in figure 4 where sound power attenuation is plotted against grid spacing $\Delta y$. The true attenuation is found by extrapolating the grid size to zero (dotted portion of the curve). As seen in Figure 4, the extrapolated numerical values for the zero Mach number case are in good agreement with the analytical values calculated from theory similar to that presented by $R$ ice in reference 1 .

The calculations shown in Figure 4 are based upon a four point numerical derivative formula rather than the two point formula used in reference 8 . As a consequence, the grid point extrapolation technique for $x$ equal 0.6 is linear. The linear extrapolation more clearly defines the answer for zero grid size, than the two point formula used in reference 8 .

\section{Finite Mach Number}

Hard wall duct. The numerical and analytical values of both the pressure and velocity are computed for the case of a one dimensional hard wall duct as was considered before (Figure 3), but with Mach numbers of 0.5 and -0.5 . The results are shown in Figures 5 and 6 . As seen in both of these figures, the agreement between the numerical and analytical results is good. In Figure 5, the positive Mach number lengthens the effective wavelength of the sound, while in figure 6 , the negative Mach number compresses the effective wavelength compared to the no-flow case (Figure 3 ).

Soft wall duct. Currently, the difference equations for the two dimensional soft walled duct with finite Mach number are being programmed and debugged. At the present time, some numerical stability problems. are occurring at low values of $\theta$ and values of $\mathrm{L} / \mathrm{H}$. near $\mathrm{l}$. We are investigating the possibility that a finer grid structure will eliminate these instabilities.

\section{Concluding Remarks}

A finite difference theory for sound propagation in a two-dimensional, soft-walled duct with uniform mean flow has been presented. The governing equations and boundary conditions are presented in difference form for both the real and imaginary pressure and velocity.

The numerical theory is shown to be in good agreement with the corresponding exact analytical results. However, because the solution matrix for the acoustical flow field is not positive definite, conventional iteration techniques cannot be used to solve the difference equations. Before the finite difference formulation can be applied to more complicated problems which require a large number of grid points, i.teration techniques or more efficient closed form solutions will have to be developed to overcome present grid size limitations.
The finite difference formulation is flexible and should be a powerful tool in studies of inlet and exhaust ducts of turbofan engines.

\section{References}

I. Rice, E. J.: Performance of Noise Suppressors for a Full-Scale Fan for Turbofan. Engines, NASA TM X-52941, Jan. 1971

2. Eversman, W.: The Effect of Mach Number on the Tuning of an Acoustic Lining in a Flow Duct. J. Acoust. Soc. Amer. 48, No. 2 (Part 1), pp. $425-428,1970$

3. Lambert, R. F.; and Tack, D. H.: Influence of Shear Flow on Sound Attenuation in a Lined Duct. J. Acoust. Soc. Amer. 38, 655-666 (1965).

4. Pridmore-Brown, D. C.: Sound Propagation in a Fluid Flowing Through an At tenuation Duct. J. Fluid Mechanics 4, 393-406 (1958).

5. Mungur, P.; and Gladwell, G. M. L.: Acoustic Wave Propagation in a Sheared Fluid Contained in a, Duct. J. Sound Vib. 9 (1), 28-48 (1969)

6. Savkar, S. D.: Propagation of Sound in Ducts with Shear Flow. Journal of Sound and Vibration $19,355-372$ (1971)

7. Shankar, P. M.: On Acoustic Refraction by Duct Shear Layers. Journal of Fluid Mechanics 47 , 81-91 (1971).

8. Baumeister, Kenneth J.: Application of Finite Difference Techniques to Noise Propagation in Jet Engine Ducts, NASA TM X-68261, Paper to be presented at ASME Winter Annual Meeting, Detroit Nov. 1973.

9. Varga, Richard S.: Matrix Iteration Analyses, Prentice-Hall, Inc., 1962

10. Morfey, C. L.: Rotating Pressure Patterns in Ducts: Their Generation and Transmission. J. Sound Vib. (1964), I, 60-87.

11. Morfey, C. L.: A Note on the Radiation Efficiency of Acoustic Duct Modes, J. Sound Vib. (1969) $9(3), 367-372$.

12. Moon, Parry; and Spencer, Domina Eberle: Field Theory for Engineers. Van Nostrand, 1961. 


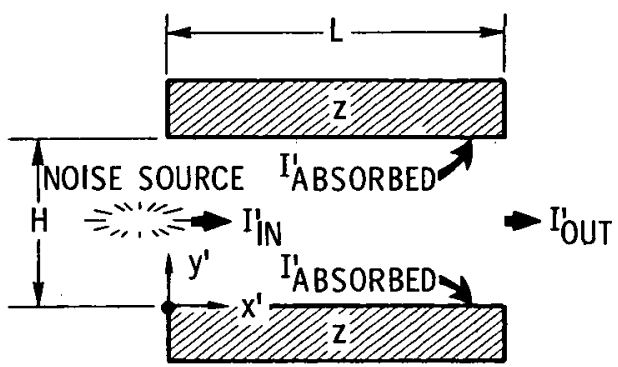

(A) DIMENSIONAL CARTESIAN COORDINATE SYSTEM, $p^{\prime}, v^{\prime}, u^{\prime}, \omega$.

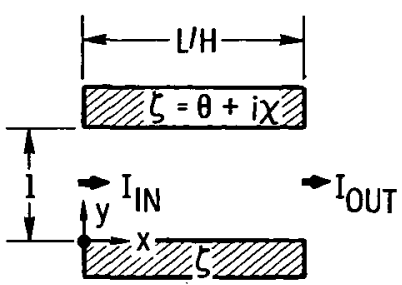

(B) DIMENSIONLESS (SCALED.) CARTESIAN COORDINATE SYSTEM, $p, u, v, \eta$.

Figure 1. - Schematic of two dimensional suppressor duct.

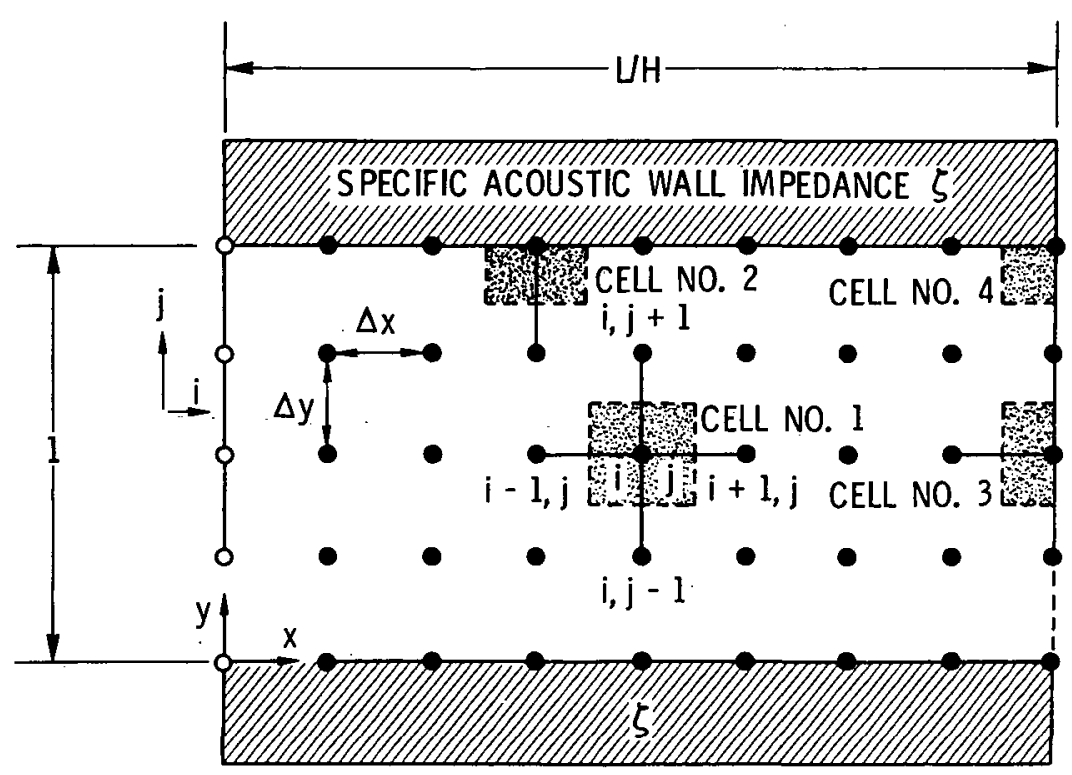

Figure 2. - Coordinate and grid point representation of two dimensional soft walled duct. 


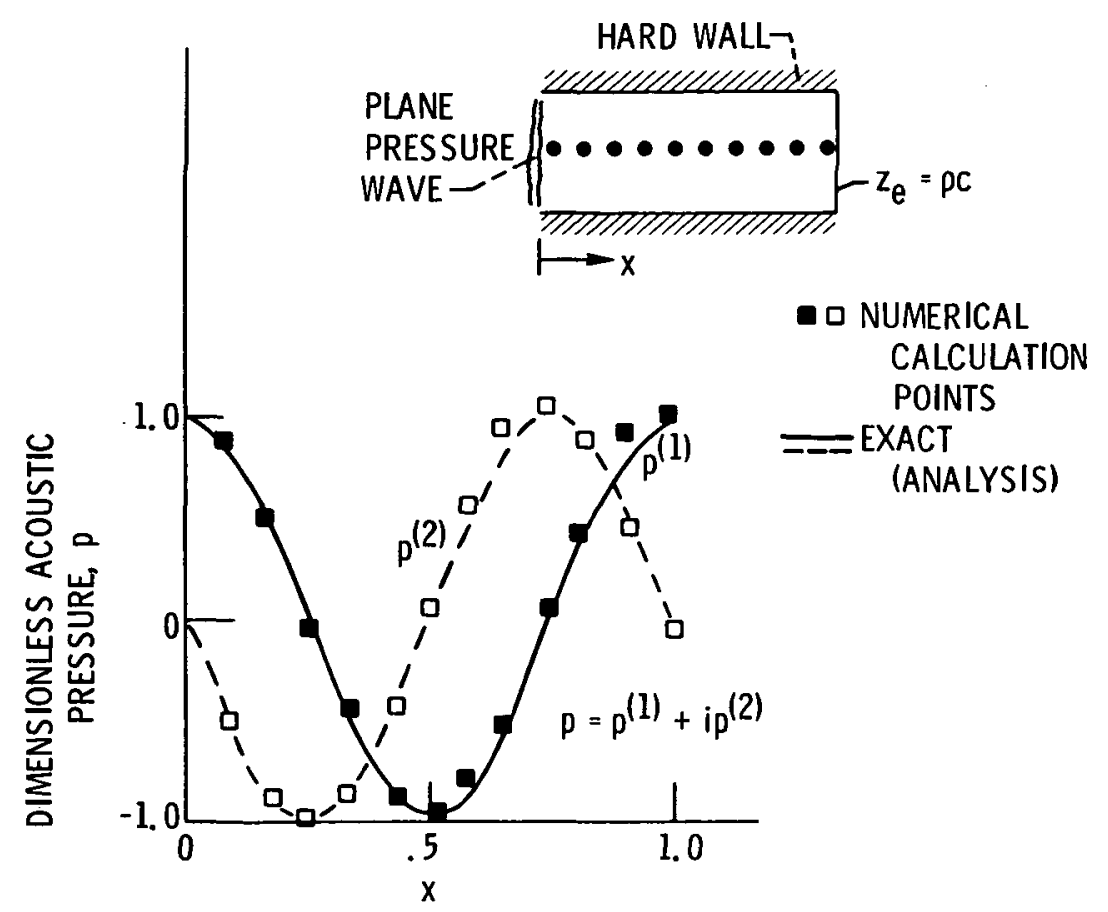

Figure 3. - Analytical and numerical pressure profiles for one dimensional sound propagation in hard wall duct for dimensionless frequency $\eta=l$ and $M=0$.

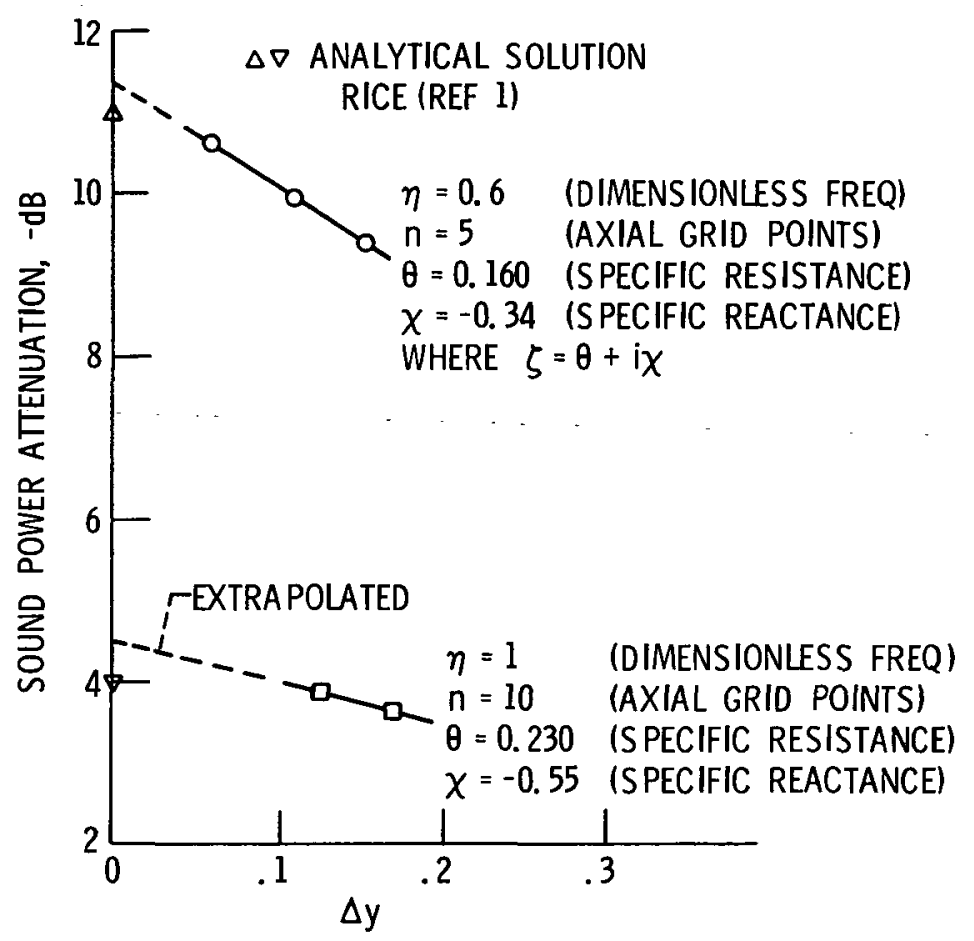

Figure 4. - Effect of spacing on attenuation in two dimensional duct for $\mathrm{UH}=0.5$ and $M=0$. 

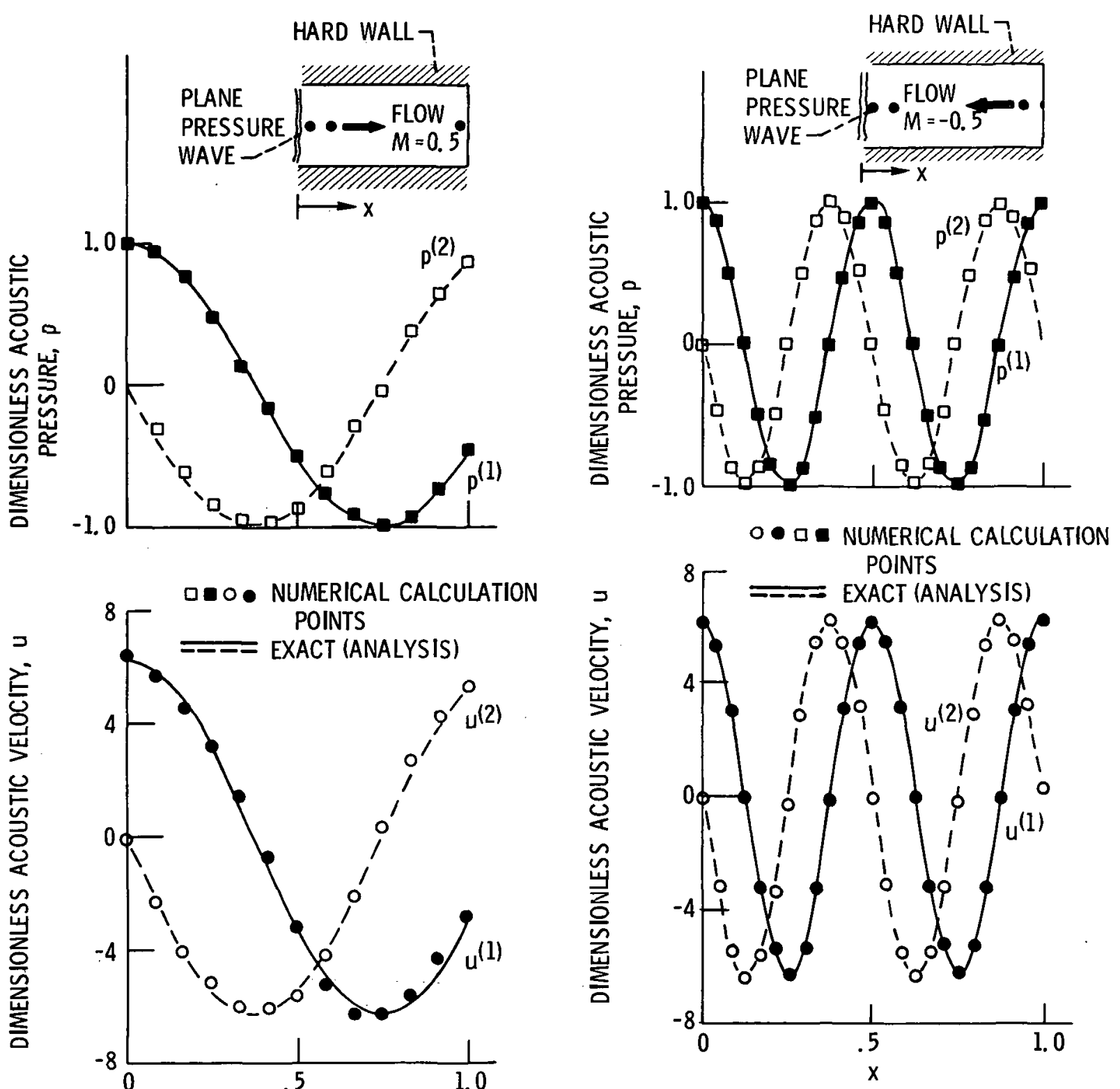

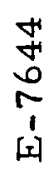

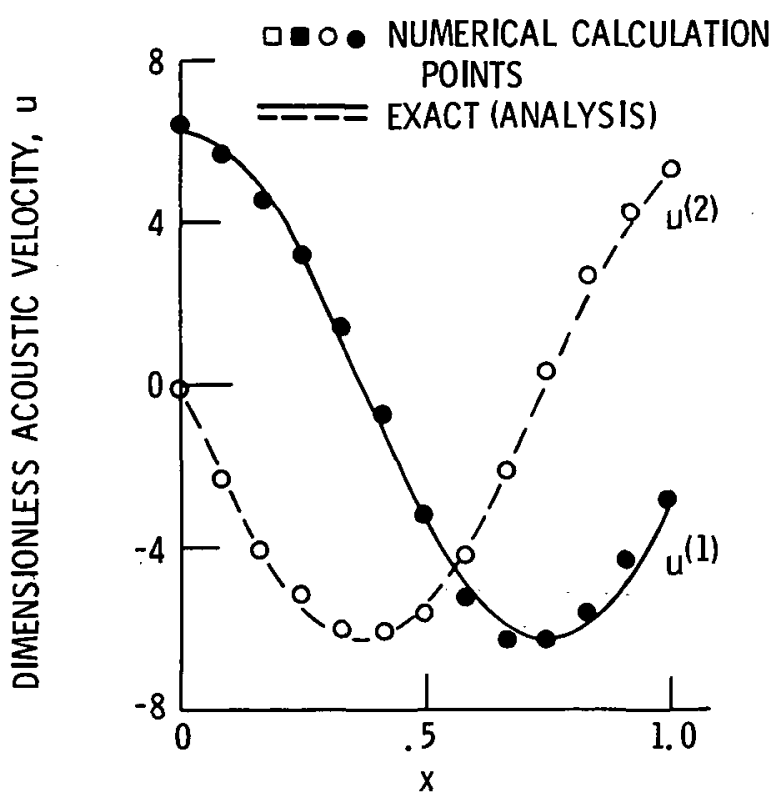

Figure 5. - Analytical and numerical pressure and velocity profiles for one dimensional sound propagation in hard wall duct for dimensionless frequency $\eta=1$ and $M=0.5$. 